\title{
Linx
}

Revue des linguistes de l'université Paris X Nanterre

64-65 | 2011

Les genres de discours vus par la grammaire

\section{Dans le genre " peut mieux faire » : le bulletin scolaire}

Salima El Hadi

\section{(2) OpenEdition}

\section{Journals}

Édition électronique

URL : http://journals.openedition.org/linx/1413

DOI : 10.4000/linx.1413

ISSN : 2118-9692

\section{Éditeur}

Presses universitaires de Paris Nanterre

\section{Édition imprimée}

Date de publication : 1 juillet 2011

Pagination : 189-206

ISSN : 0246-8743

\section{Référence électronique}

Salima El Hadi, « Dans le genre « peut mieux faire » : le bulletin scolaire », Linx [En ligne], 64-65 | 2011,

mis en ligne le 01 juillet 2014, consulté le 19 avril 2019. URL : http://journals.openedition.org/linx/1413 ; DOl : 10.4000/linx.1413 


\title{
Dans le genre « peut mieux faire » : le bulletin scolaire
}

\author{
Salima E1 Hadi \\ Université de Bourgogne \\ Collège L. Pergaud - Pierrefontaine-les-Varans (25)
}

Dans le cadre de la réflexion sur les rapports entre genre et grammaire nous avons choisi de nous intéresser, pour les subtilités d'analyse qu'il permet, au bulletin scolaire. La partie linguistique du bulletin sur laquelle nous porterons principalement notre attention se présente comme un commentaire évaluatif ou appréciatif censé rendre compte de la valeur scolaire du travail fourni par un individu clairement désigné. Ce commentaire est posé en vis-à-vis d’un ensemble de données chiffrées, les notes, qu'il doit éclairer en en fournissant une interprétation.

En tant que discours contraint inséré dans une situation sociale de communication assez bien définie, le bulletin correspond à ce que D. Maingueneau (2004) définit comme une routine, routine que suffit d'ailleurs à évoquer cette phrase particulièrement emblématique du bulletin qu'est le fameux « Peut mieux faire. ». Dans une classification des genres, au sens où l'entend F. Rastier (2001), le bulletin scolaire relèverait plus généralement du «champ générique » des écrits d'évaluation, lequel découlerait naturellement $\mathrm{du}$ "discours » éducatif. Le bulletin scolaire ne semble pas lui-même déclinable en «sous-genres » même s’il peut présenter des variantes (appréciations manuscrites / tapuscrites, subdivisions de l'appréciation) dont nous reparlerons. Mais cette notion de routine que nous invoquons et qui semble si rassurante par la stabilité qu'elle implique presque par définition, ne doit pas masquer la complexité des faits de langue que l'on est susceptible d'y observer. En effet, ceux-ci ne donnent pas toujours au genre de discours l'aspect d'un simple patron répondant à un ensemble de conventions stables, et révèlent au contraire une aptitude à 
l'hétérogénéité qui nécessite d'approfondir l'approche de ces schémas routiniers. Loin de chercher à remettre en question leur pertinence, il s'agira surtout d'en révéler l'intéressante subtilité, subtilité qui nous renseigne autant sur le système général de la langue que sur les pratiques sociales, historiquement et géographiquement marquées ${ }^{1}$, qui sous-tendent ses usages spécifiques. Si nous évoquons cette potentielle hétérogénéité du genre de discours, c'est parce que celle-ci s'impose d'elle-même dans le bulletin scolaire dès lors que l'on envisage la question du destinataire, qui, loin d'être d'ordre purement pragmatique, est directement appelée et même légitimée par l'examen des faits de langue.

Nous avons consulté de nombreux bulletins, puis nous avons examiné plus précisément une soixantaine d'entre eux (soit environ 650 appréciations) avant de choisir de resserrer notre étude autour de dix-huit exemplaires édités en 2010-2011, que nous avons considéré comme représentatifs. Ces dix-huit bulletins, qui proviennent de cinq établissements différents ${ }^{2}$, constitueront notre corpus de référence. Ils totalisent 196 commentaires appréciatifs rédigés par 120 locuteurs. Ce corpus de référence, outre sa représentativité, permet, par son caractère restreint, une visualisation rapide des proportions, quantifiables en pourcentages. Nous reconnaissons d'emblée que les résultats obtenus ne peuvent prétendre qu'à une valeur relative, et ce en raison même de la restriction que nous évoquions. Il s'agit surtout de mettre au jour des tendances linguistiques autorisant des observations sur les relations entre les éléments grammaticaux et la place sociale des bulletins scolaires. Dans cette perspective, nous nous attacherons d'abord à mettre en évidence les faits de langue les plus représentatifs du fonctionnement routinier du genre. Sans quitter le champ des faits de langue, nous verrons ensuite comment ceux-ci peuvent interroger la notion même de routine en en révélant la paradoxale complexité. Afin de mener à bien cette tâche dans le respect de la perspective comparatiste qu'impose la description de genres qui se définissent par effet de rivalité et de concurrence, nous avons tenu à convoquer le genre voisin du livre d'or qui, en tant que commentaire évaluatif orienté vers la même brièveté que le bulletin scolaire, nous offrira quelquefois de précieux contrepoints ${ }^{3}$.

\footnotetext{
${ }^{1}$ Par cette remarque, nous entendons insister sur le fait que le schéma que nous présentons n'a qu'une valeur ponctuelle. Les faits de langue sélectionnés par le genre du bulletin représentent en effet la photographie d'une époque et d'un espace social bien définis. Une approche plus diachronique ou étendue à d'autres espaces culturels permettrait des comparaisons qui ouvriraient sans doute une perspective encore plus intéressante au travail sur les rapports entre genre et grammaire.

2 Ajoutons, pour être plus précis, que ces établissements sont représentés par des lycées d'enseignement général et technologique et des lycées professionnels.

${ }^{3}$ Nous avons choisi, pour sa facilité d'accès pour le lecteur, un livre d'or numérique consultable sur le site Internet «Contefleur », qui propose des jeux et des activités diverses en rapport avec la nature. Pour plus de détails, suivre le lien www.contefleur.fr/images/livredor.htm.
} 


\section{Les marques grammaticales les plus routinières du genre}

En cherchant à mettre en évidence les caractéristiques les plus remarquables du genre du bulletin, nous verrons que celui-ci tend à générer de l'impersonnel. Nous évoquerons cet aspect à travers la question de l'ellipse du sujet dont nous discuterons la valeur prototypique. Nous le verrons également à travers la référence des démonstratifs ce et cela, et l'étude des énoncés averbaux organisés autour de groupes nominaux expansés. Si au moment d'aborder la question du lexique, nous insisterons davantage sur le figement des syntagmes observés, nous verrons que ce lexique contribue lui aussi à générer de l'impersonnel, en dispensant le locuteur de produire un propos véritablement original.

\subsection{L'absence de sujet : la marque prototypique du genre?}

Nous évoquions dans le titre de notre contribution la phrase emblématique du genre qu'est «Peut mieux faire.». Celle-ci nous amène naturellement à poser la question de l'absence de sujet.

Dans les exemplaires que nous avons consultés comme dans ceux qui constituent notre corpus, nous n'avons trouvé que de rares exemples d'absence de sujet dans des phrases répondant par ailleurs au schéma verbe + complément. Dans notre corpus de référence, nous n'en avons isolé que 3 (au présent et au passé composé), ce qui ne représente que $1,5 \%$ de l'ensemble des appréciations. Nous les reproduisons ci-dessous en (1).

(1a) Travaille avec sérieux et méthode.

(1b) N'a pas baissé les bras et a gagné en assurance.

(1c) Sait très bien décrire et comparer à l'écrit.

L'ellipse du sujet pourrait s'expliquer par le fait que celui-ci, trop constamment référentiel à l'élève dont on parle, serait considéré comme inutile de par sa grande saillance ${ }^{4}$. La prise en compte de cette dernière notion serait elle-même incitée par une contrainte purement formelle qui limite le texte à 300 caractères environ et invite ainsi l'auteur du commentaire à supprimer toute mention inutile. Il faut noter que cette limitation du texte reste valable dans le cas d'appréciations notées à la main : en effet, le bulletin vierge qui est alors édité respecte toujours le format $\mathrm{A} 4$, ce qui limite le champ d'intervention de chaque scripteur à trois demi-lignes environ. Nous retrouvons la même brièveté du propos dans les appréciations du livre d'or numérique qui nous sert de point de comparaison et qui n'imposait pourtant aucune limitation de caractères. Pour justifier la concision du propos dans ce contexte, on peut alors faire valoir une sorte d'effet mimétique par rapport au support papier traditionnel qui interdit socialement que l'on occupe une place indue dans un cahier ou un livre de

\footnotetext{
${ }^{4}$ Cette saillance, construite et limitée par le genre de discours dans sa structuration matérielle, est comparable aux énoncés visibles par exemple sur les bouteilles de soda « se boit très frais » ou encore sur les indicateurs de chemin de fer «ne prend pas de voyageur au départ d'Orléans ».
} 
quelques dizaines de pages. Quoi qu'il en soit sur cette question, nous noterons que la restriction quantitative du texte n'a pas amené à pratiquer l'absence de sujet. Mais revenons au bulletin. Si l'on s'en tient aux chiffres, on tendra à considérer que l'absence de sujet ne peut guère être considérée comme une marque distinctive du genre. Pourtant, il semble que la question de l'ellipse du sujet ne puisse pas être évacuée aussi rapidement. Afin d'étayer ce point de vue, nous aimerions faire référence à la réalisation d'un exercice scolaire qui impliquait la rédaction d'un bulletin ${ }^{5}$. Nous avons demandé aux élèves engagés dans cette tâche d'imaginer le bulletin scolaire que les différents maitres intervenus auprès de « $\mathrm{M}$. Jourdain » auraient pu rédiger pour rendre compte de ses performances. Certains élèves ont alors spontanément pratiqué l'absence de sujet dans des phrases auxquelles ils ont conservé un verbe et au moins un complément. Pourtant, le faible nombre d'occurrences que nous avons relevé dans les bulletins réels édités actuellement autorise à penser que la pratique de l'absence de sujet n'a pas été inspirée à ces élèves par la lecture de leurs propres bulletins. L'appropriation de cette pratique peut paraitre d'autant plus énigmatique que rien dans la consigne de l'exercice ne les incitait à éluder le sujet et que l'exemple illustratif choisi par les concepteurs du manuel n'exploitait pas non plus cette possibilité. On constate donc que si l'ellipse du sujet ne constitue pas une pratique particulièrement remarquable ou courante à l'heure actuelle, elle reste, virtuellement et presque symboliquement, une marque du genre ${ }^{6}$. Si nous ne chercherons pas à connaitre ici les raisons de cette imprégnation symbolique auprès des élèves, nous pouvons tenter d'expliquer pourquoi la séquence sujet vide + verbe + complément est aujourd'hui rarement présente dans les bulletins scolaires. Cette pratique linguistique est peut-être désormais perçue comme une forme assez discourtoise du fait de la dépersonnalisation, si l'on peut dire, qui l'accompagne 7 . A une époque devenue très sensible à la question du respect de l'élève, l'absence de sujet grammatical prend le risque d'être assimilée à un véritable dénigrement de l'individu.

\subsection{Référence de ce / cela}

Les pronoms ce et cela apparaissent régulièrement dans les commentaires de bulletins, la plupart du temps dans les tours lexicalisés «c'est bien », «c'est très bien »

\footnotetext{
${ }^{5}$ Cet exercice a été réalisé par des élèves de cinquième du collège de Pierrefontaine-les-Varans (Doubs). Le détail de la consigne figure dans le manuel Jardin des lettres, $5^{\mathrm{e}}$ (Magnard, 2010 : 185).

${ }^{6}$ On peut d'ailleurs remarquer, nous semble-t-il avec un certain profit, que lorsque le bulletin scolaire est pastiché dans un but humoristique, l'absence de sujet est alors largement pratiquée comme tendent à le montrer les multiples exemplaires qu'une simple recherche sur Internet permettra de se procurer.

${ }^{7}$ Si la notion de sujet vide peut permettre d'expliquer le ressenti potentiellement négatif du destinataire, elle ne suffit cependant pas à rendre compte d'une éventuelle réaction « en amont » de la part du locuteur. Celui-ci peut être amené à éviter cette structure non pas tant en raison de ses caractéristiques linguistiques intrinsèques que parce qu'il craint qu'elle ne soit perçue comme trop prototypique du genre. L'évitement de la structure s'expliquerait alors par le fait que le locuteur, conscient de ne faire aucun effort d'originalité, pourrait se reprocher d'avoir négligé la spécificité individuelle de l'élève dont il parle et auquel il parle.
} 
ou «c'est insuffisant ». Certaines occurrences ne posent pas de problème particulier d'interprétation. On peut citer à cet endroit les exemples (2a) et (2b) suivants :

(2a) Des résultats en progression et devenus satisfaisants, c'est bien.

(2b) Les résultats et l'attitude sont toujours excellents et cela en toute modestie.

$\mathrm{Ce}$ et cela reprennent ici de manière anaphorique des notions récemment évoquées (relatives aux résultats) et qui n'ont plus besoin d'être spécifiées. Pourtant, dans l'exemple qui suit, l'interprétation anaphorique s'avère déjà plus délicate :

(3) Assez bon travail, mais cela reste fragile cependant.

Ici, on peut bien sûr défendre l'idée que «cela» est une reprise du contenu sémantique du nom «travail» mais le pronom démonstratif crée une zone d'indéfinition qui rend l'interprétation beaucoup plus approximative que dans un énoncé du type «Assez bon travail, mais qui reste fragile cependant. ».

Certaines occurrences du pronom démonstratif posent quant à elles un réel problème. C'est le cas des exemples (4a) et (4b) :

(4a) C'est toujours très bien.

(4b) C'est très bien. Compliments.

Il faut préciser que ces deux extraits ne constituent pas des citations tronquées mais bien l'intégralité du commentaire des professeurs respectivement impliqués. Dans une telle perspective, l'interprétation anaphorique devient tout à fait impossible. On peut dès lors légitimement se demander à quoi réfère le pronom $c e$ dans une situation où la distance spatiale et temporelle devrait abolir l'interprétation déictique. Certes, la note chiffrée correspondant aux travaux de l'élève vient partiellement éclairer la signification du pronom. Toujours est-il que pour interpréter ce type d'exemple, il faut pouvoir admettre un certain degré de connivence entre les interlocuteurs ou reconnaitre l'existence d'une situation saillante susceptible de permettre à l'interlocuteur d'interpréter le message qu'on lui adresse en se représentant un objet qu'on ne lui désigne pas explicitement dans la phrase. De telles incertitudes sur l'interprétation référentielle révèlent peut-être un des aspects essentiels du bulletin qui serait de générer de l'impersonnel.

\subsection{Les noms ou groupes nominaux expansés}

Dans les exemples que nous avons analysés, la caractéristique la plus remarquable du genre repose sur la production d'énoncés averbaux (Lefeuvre, 1999) se présentant comme des phrases organisées autour de noms ou de groupes nominaux. Nous remarquerons que ce dernier trait distinctif est aussi bien représenté dans le bulletin que dans le livre d'or. L'autre domaine que nous aborderons sera celui du lexique, notablement concerné en tant qu'il est toujours à l'avant-garde des procédés d'écriture.

Nous l'avons dit, les séquences nominales sont particulièrement représentatives du bulletin scolaire. On observe une récurrence très forte de la double configuration suivante (présente à hauteur de $82 \%$ dans notre corpus) : 
(i) Nom expansé sans déterminant

(5a) Trimestre satisfaisant.

(5b) Excellent travail.

(5c) Résultats satisfaisants.

(5d) Bon travail (...)

(5e) Bon trimestre (...)

(5f) Élève agréable (...)

(ii) Groupe nominaux précédés d'un article indéfini ou partitif :

(6) Des capacités, du sérieux, du travail.

Devant ces groupes nominaux précédés d’un déterminant, on peut généralement restituer un présentatif du type il y a / c'est ${ }^{8}$.

Les groupes nominaux sont en général expansés de deux manières :

(i) à l'aide d'un adjectif placé à un degré d'intensité.

Dans ce cas, les possibilités de variation de l'adverbe d'intensité sont plutôt limitées et se bornent globalement à l'utilisation de très, trop et assez: Dans le livre d'or (7a-c) qui nous sert de point de comparaison, nous retrouvons une utilisation assez fréquente de ces groupes nominaux à valeur descriptive dans des phrases averbales réellement très proches de celles que l'on pourrait trouver dans un bulletin scolaire. On notera également dans les exemples qui suivent l'utilisation des degrés d'intensité :

(7a) Trop joli votre site.

(7b) Très beau travail (...)

(7c) Très beau site, intéressant, pertinent, pédagogique.

(ii) à l'aide d'une proposition subordonnée relative adjective qui joue ici pleinement son rôle d'expansion nominale :

(8a) Des résultats globalement satisfaisants qui peuvent progresser.

(8b) Elève sérieux qui a pris confiance en lui.

On remarquera cependant que les relatives adjectives sont beaucoup moins présentes dans le livre d'or et que lorsqu'elles le sont, elles s'insèrent plus volontiers dans l'économie de la phrase complexe :

(9) Je vous remercie de tous ces jeux qui ne me lassent pas du tout et qui me rappellent toute mon enfance, j’y passe des heures...

Si on s'intéresse maintenant aux caractéristiques des phrases verbales telles qu'elles se présentent dans le bulletin, on s'aperçoit alors qu'elles ne décrivent

\footnotetext{
8 Rappelons à cet endroit que l'emploi du démonstratif pose un problème de référence que nous avons évoqué plus haut.
} 
qu'exceptionnellement un véritable procès dynamique. Les verbes les plus utilisés sont en effet attributifs et ils s'insèrent dans des phrases qui ont la même visée descriptive ou caractérisante que les expansions nominales :

(10a) L'investissement a été sérieux tout au long du trimestre.

(10b) (...) cela reste fragile cependant.

(10c) (...) votre travail est resté mesuré.

(10d) (...) cela reste tout juste suffisant.

(10e) (...) cela reste assez modeste.

Ces structures attributives à valeur descriptive sont également bien représentées dans le genre du livre d'or :

(11a) Ce minicolor est très bien et amusant.

(11b) C'est très chonette.

(11c) Votre site est vraiment bien.

(11d) (...) ils sont sympas et super bien faits.

La comparaison entre le bulletin et le livre d'or permet de montrer que l'appréciation se présente de manière assez stable comme un contenu descriptif et cherche à poser un constat, une donnée finie, un résultat. La forte prévalence des groupes nominaux chargés de présenter des données objectivables peut être perçue comme une manière de générer de l'impersonnel : le locuteur s'absente, si l'on peut dire, des constats qu'il pose tout comme il en écarte l'interlocuteur concerné par ceuxci. Il faut noter que cette dernière remarque s'applique davantage au bulletin qu'au livre d'or comme nous le verrons plus loin lorsque sera abordée la question du destinataire et la place accordée à la subjectivité de l'énonciateur.

\subsection{Le lexique}

L'étude du lexique permet d'approfondir l'analyse des groupes nominaux expansés car elle amène à souligner le caractère figé de ces groupes qui fonctionnent le plus souvent comme des étiquettes nominales.

Le constat le plus évident que l'on puisse faire concerne l'aspect restreint et répétitif du lexique et la faible originalité des syntagmes obtenus de ce fait en guise de commentaires évaluatifs. On peut tout d'abord expliquer cette restriction par un simple argument logique : définir un thème ou s'inscrire dans un domaine d'activités amène le locuteur à restreindre son lexique autour du thème ou du domaine en question. Mais on peut également faire valoir un argument d'ordre social qui prend en considération le statut du scripteur : l'enseignant fonctionnaire qui rédige des appréciations se doit de respecter la norme sociale et académique qu'il estime correspondre à sa fonction. Il faut cependant noter que le lexique employé dans les bulletins est considérablement plus limité que l'éventail linguistique qu’un professeur est tacitement libre d'utiliser. 
Parmi les termes fréquemment employés dans notre corpus, on peut citer les noms et adjectifs suivants : participation, fragile, efforts, sérieux, travail, matière, trimestre, bavardage, attentif, convenable, ensemble, insuffisant, satisfaisant. Si la limitation du lexique est assez naturelle pour les raisons que nous avons évoquées, ce qui interpelle davantage c'est que, parmi les termes utilisés, certains ne sont pas directement appelés ou justifiés par le contexte scolaire. À l'inverse des noms travail ou matière, les termes fragile, ensemble, investir, convenable ou approfondir apparaissent avec une assez grande régularité sans qu'aucune nécessité ne vienne pourtant l'expliquer. Tout se passe comme si le locuteur employait ces mots sans passer en revue les différentes acceptions qu'ils comportent dans la langue mais en se référant au contenu sémantique des syntagmes relativement figés dans lesquels ils figurent régulièrement sur les bulletins et qui lui servent de référents au moment de rédiger son texte. Cette tendance au figement d'expressions composées (Moreau, 1992) est facilement perceptible lorsqu'on procède à quelques tests de commutation : ensemble insuffisant ne s'oppose pas à ensemble suffisant alors que rien n'y fait obstacle du point de vue sémantique. De la même manière, trimestre satisfaisant ne trouve pas son contradictoire dans trimestre insatisfaisant auquel on préfèrerait probablement la formule trimestre insuffisant. Ces syntagmes figés tendent à donner l'image d'une appréciation composée d'étiquettes qui limitent l'originalité de la production linguistique du locuteur. Le travail de rédaction des appréciations consisterait principalement en la sélection de ces étiquettes consacrées par l'usage et / ou mises à la disposition du professeur par l'expérience. Ceci finit par donner l'image d'une langue extrêmement codifiée qui justifie presque à elle seule l'évocation de la notion de genre dans laquelle nous cherchons à nous inscrire. Nous n'avons d'ailleurs pas constaté d'écart stylistique notable chez certains locuteurs. Le propos est donc marqué par une grande prédictibilité et, tout au moins dans les exemplaires que nous avons consultés, par une fréquence de transgression parfaitement nulle. Cette transgression est pourtant toujours envisageable, y compris dans les genres les plus contraints (Branca, 1987).

Il est à noter que ce goût pour les étiquettes nominales ne s'explique pas seulement par l'intention essentiellement descriptive que l'on accorde à l'appréciation et que nous avons évoquée précédemment mais renvoie à une posture globale assimilable à une recherche constante du figement : ainsi, le syntagme « félicitations du conseil de classe», qui n'a pas de fonction descriptive mais plutôt une valeur performative (Krazem, 2012) au moment où il est découvert par le jeune lecteur, est-il révélateur d'une certaine complaisance vis-à-vis d'une forme de rigidité linguistique. La langue employée dans le bulletin chercherait donc à créer des séquences linguistiques préconstruites qui, in fine, dispenseraient le locuteur de véritablement choisir ses mots. Cette intention est d'ailleurs rendue particulièrement manifeste par la création de l'étiquette électronique «félicitations » présente dans deux bulletins de notre corpus, qui réduit l'effort linguistique à un simple acte, celui de cocher une case?

L'étude du lexique confirme donc l'idée que le bulletin scolaire cherche à produire une langue capable de créer des catégories objectives et stables d'évaluation. Une sorte

\footnotetext{
${ }^{9}$ Dans les deux bulletins que nous évoquons, le professeur principal avait la possibilité, à l'issue ou au cours du conseil de classe, de cliquer sur une case numérique qui permettait de faire apparaître automatiquement la mention « félicitations » sur les bulletins édités.
} 
d'idéal pourrait presque être représenté par un professeur qui sélectionnerait, grâce à l'outil informatique, les étiquettes nominales préformatées qui, par leur contenu descriptif, conviendraient le mieux à la valeur scolaire de l'individu concerné ${ }^{10}$. Cette idée d'une évaluation effectuée par des catégories préétablies pose implicitement la question de l'implication du sujet. Le fait d'utiliser des étiquettes nominales figées semble en effet protéger le locuteur qui ne se risque qu'à avancer le contenu sémantique officiel que l'institution a reconnu à un ensemble de termes bien définis. Peut-on dès lors commencer à parler de langage spécialisé ? En effet, en dehors du contexte du bulletin scolaire, qu'est-ce qu'un « ensemble insuffisant » ? Cette tendance au jargon peut également être perçue comme une stratégie linguistique visant à générer de l'impersonnel. En s'exprimant à travers les termes officiels de l'institution, le locuteur n'utilise plus la langue comme un moyen d'expression individuel et original mais cherche plutôt à rejoindre un certain état de la langue.

À ce premier constat s'ajoute le fait que le lexique est régulièrement engagé dans la production de tournures euphémistiques, ou si l'on préfère diplomates, dans lesquelles on perçoit la réserve de celui qui juge tout en se gardant de souligner trop fortement les réalités, en particulier lorsque celles-ci sont négatives. On peut bien sûr évoquer l'exemple très connu de manvais remplacé par insuffisant ou fragile afin d'éviter d'avoir recours à l'adjectif faible, jugé sans doute plus dévalorisant. On assiste ainsi à la production d'un discours que l'on pourrait qualifier d'anti-polémique qui, nous le verrons, trouve une continuité logique à travers le désengagement de l'interlocuteur. La notion de discours anti-polémique se justifie particulièrement dès lors qu'on envisage les énoncés de bulletins du point de vue pragmatique. En effet, alors que le discours polémique cherche, par diverses stratégies, à engager avec force la responsabilité de l'interlocuteur, le discours anti-polémique tel qu'il est à l'œuvre dans les bulletins scolaires, tend à procéder de manière strictement inverse, tant en affaiblissant la position de sujet engagé du locuteur qu'en évitant assez soigneusement de mettre en cause l'allocutaire. Finalement, c'est l'ensemble de la «scène d'énonciation » qui se trouve dépassionnée, dans un savant effort pour regagner le terrain de la neutralité ${ }^{11}$.

\section{Une routine moins schématique qu'il n'y paraît : complexité de la relation énonciative}

Le genre du bulletin est marqué par le retranchement énonciatif du locuteur qui définit lui-même un rapport au destinataire fondé sur la réserve et la prudence. Ce destinataire n'a d'ailleurs pas qu'un visage : au-delà du seul allocutaire adressé que représente l'élève gravitent des destinataires indirects, que ceux-ci soient facilement identifiables par des marques particulièrement explicites ou appréhendables par une prise en considération de l'ensemble de la situation d'énonciation. Les travaux d'Alain Rabatel (2010) nous apporteront une aide précieuse dans l'analyse de cette situation complexe liée à la question du destinataire.

\footnotetext{
${ }^{10}$ Nous tenons à préciser qu'il ne s'agit là que d'une extrapolation de notre part puisqu'une telle option ne semble pas exister à l'heure actuelle.

${ }^{11}$ Voir P. Charaudeau, D. Maingueneau, 2002 : 437-438.
} 


\subsection{Désengagement du locuteur : stratégies d'évitement de la première personne}

Il est tout à fait frappant de voir à quel point, dans le bulletin, les locuteurs prennent soin d'éviter de se désigner par le pronom je ou par toute autre marque de la première personne. Ainsi, sur les 196 appréciations dont notre corpus est constitué, seules trois utilisent une marque de la première personne, soit 1,5\% de la totalité ${ }^{2}$. Dans le genre du livre d'or, la première personne apparait avec une régularité qui marque significativement l'écart avec le genre du bulletin. Dans l'exemplaire que nous avons examiné, $57 \%$ des évaluations comportaient une marque de la première personne et 20,5\% exhibaient même une posture assumée d'appréciation dans des tours tels que « je trouve que », «je pense », «j’aime » qui montrent bien que la subjectivité est licite. En réalité, elle est même explicitement recherchée dans ce genre de discours: l'initiateur du livre d'or a en effet directement sollicité l'opinion personnelle des scripteurs auxquels il semble offrir une véritable tribune de libre expression. Les bulletins que nous avons analysés permettent quant à eux de mettre à jour deux principales stratégies d'évitement de la première personne :

(i) Le locuteur utilise pour se désigner une marque de la troisième personne.

Dans l'exemple (12), le locuteur utilise ainsi la grande flexibilité du pronom indéfini on, surtout si on considère ses inscriptions génériques (Bouquet, 2007), pour se désigner lui-même au milieu d'un ensemble collégial plus vaste quoique mal délimité :

(12) On ne peut que l'encourager à poursuivre au troisième trimestre.

Dans l'exemple (13) qui suit, le locuteur se désigne quant à lui par le groupe nominal «le professeur» par lequel il semble rappeler qu'il ne juge qu'en vertu des pouvoirs que lui confère son statut:

(13) Il faut appliquer les méthodes préconisées par le professeur.

(ii) Le locuteur utilise la forme passive afin d'effacer les marques de sa présence subjective.

On peut ainsi lire dans l'un des exemplaires de notre corpus «Vous avez tenu compte des conseils donnés. » alors que la relation directe et personnelle qui lie le professeur et l'élève permettrait légitimement d'attendre un «Vous avez tenu compte des conseils que je vous ai donnés. » Une autre appréciation témoigne du même type de désengagement : le professeur écrit «Les efforts attendus » alors que l'on pourrait attendre, pour les raisons que nous venons d'évoquer, «Les efforts que j’attendais ».

Si le désengagement du locuteur semble bien attesté, il doit, pour trouver pleinement son sens, être mis en perspective grâce à l'analyse de la relation au destinataire.

\footnotetext{
12 C'est finalement ce que laissait déjà pressentir l'orientation que nous avions prise dans la première partie de cette contribution lorsque nous évoquions les tendances du genre à l'impersonnel.
} 


\subsection{La question du destinataire : incertitudes liées à situation de communication}

Comme nous l'évoquions plus haut, la question de la relation énonciative ne saurait être envisagée de manière monolithique, ce que signale d'ailleurs la position parfaitement éclairante d'A. Rabatel (2010) qui rappelle que la question du destinataire ne peut se résumer systématiquement à une interaction strictement bilatérale. Le destinataire n'est pas « un », il ne doit pas non plus être envisagé comme un ensemble d'instances disparates mais comme une entité stratifiée, multiple certes, mais aussi complémentaire et simultanée. Il nous faut donc tenir compte de ces acquis au moment d'analyser le genre qui nous intéresse. Si le bulletin s'adresse directement au seul élève, il s'adresse aussi indirectement et simultanément aux parents et à l'administration. En marge de ces trois destinataires, il nous faudra également envisager l'influence des collègues ou des pairs qui agissent comme un quatrième destinataire, susceptible d'influencer les propos tenus par le locuteur. Même si cette influence est plus difficile à appréhender ou à évaluer, elle n'amène pas nécessairement à quitter le champ des marques linguistiques, comme nous tenterons de le montrer.

\subsubsection{Destinataire direct : le professeur s'adresse à l'élève}

2.2.1.1. Les marques explicites d'une relation d'interlocution

L'adresse directe à l'élève maintient ou non l'usage établi entre les interlocuteurs : on retrouve, dans notre corpus, une forte prévalence du vous présent dans $85 \%$ des appréciations présentant une marque de la deuxième personne. Cette proportion n'est cependant pas révélatrice de l'emploi du vouvoiement dans les classes à l'heure actuelle. Il semble plutôt que, dans un grand nombre de cas, le vouvoiement soit spontanément substitué au tutoiement par les enseignants afin de s'adapter aux attentes tacites générées par la production d'un écrit officiel. Les exemples (14) montrent en tout cas que les deux modes d'adresse peuvent exister sur un même bulletin :

(14a) Davantage d'application dans les devoirs à la maison vous fera encore progresser.

(14b) (...) tu n'en retireras que des bienfaits.

Plus rarement, le professeur utilise l'apostrophe et interpelle l'élève par son prénom d'une manière qui rappelle l'encouragement appuyé que l'on peut donner à l'oral :

(15) Continuez l'année prochaine, Jean-Baptiste.

\subsubsection{Le relatif effacement de l'interlocuteur}

Même si nous avons pu repérer des marques explicites d'une relation d'interlocution, l'examen des bulletins montre que la notion de professeur s'adressant à l'élève peut et doit largement être nuancée.

Avant d'entamer réellement notre propos sur ce sujet, notons que l'appréciation se compose souvent de deux parties distinctes : la première, évaluative, pose un 
constat à un moment précis, constat qui, ne l'oublions pas, prend acte des notes obtenues par l'élève. La seconde, quand elle existe, est assimilable à une exhortation ou à une injonction plus ou moins forte. Certains bulletins établissent d'ailleurs une distinction explicite entre le constat d'appréciation, logiquement tourné vers le passé, et le conseil orienté vers le futur et dont la valeur prescriptive peut donner lieu à l'emploi de l'infinitif (16), à la manière d'une séquence procédurale que J-M. Adam (2010) analyse comme des descriptions d'actions ${ }^{13}$ :

(16) Très bon trimestre, poursuivre ainsi.

L'appréciation du bulletin scolaire se présente alors globalement comme un genre à séquences descriptives : [notes chiffrées + description-évaluation] + [description d'action].

L'étude du propos injonctif dans la partie de l'appréciation qui sert à conseiller ou à prescrire nous livre des informations intéressantes sur les stratégies d'effacement des marques de la subjectivité. Voici l'état des lieux que nous pouvons établir sur cette question :

(i) Le mode impératif est assez bien représenté ce qui ne doit pas nous étonner. On peut en effet lui conférer une sorte de légitimité pédagogique de par sa clarté et son caractère direct, frontal.

(17a) Soyezplus présent et actif.

(17b) Soyez attentif et apprenez:

Il faut cependant préciser que les deux exemples (17a) et (17b) constituent des injonctions fermes assez rares. Plus souvent, le mode impératif est utilisé dans des exhortations positives qui ont essentiellement une valeur d'encouragement (c'est même le cas de 76\% des impératifs rencontrés dans notre corpus) :

(18a) Continuez ainsi.

(18b) Poursuivez vos efforts.

(18c) Poursuivez ainsi.

(ii) En marge de cette utilisation assez naturelle de l'impératif, on observe des procédés d'atténuation du discours injonctif qui viennent largement concurrencer le mode impératif.

-Présence très fréquente du tour impersonnel il faut qui représente à lui seul $34 \%$ de tous les propos injonctifs présents dans notre corpus. Dans ce cas, la référence du « sujet» sémantique vide de l'infinitif est reconstruite grâce aux propriétés du genre qui, comme nous l'avons évoqué dans la première partie, tend à poser comme référence par défaut la personne de l'élève et autorise ainsi une stratégie d'allusion permanente :

13 L'emploi d'infinitifs de prescription est davantage repérable dans les modèles préformatés de bulletins qui distinguent formellement l'appréciation et le conseil. L'existence de cette scission justifie d'ailleurs que la question de l'infinitif fasse l'objet d'un traitement à part, sans lien avec les groupes nominaux expansés que nous avons évoqués antérieurement. 
(19a) Il faut travailler davantage.

(19b) (..) il faut davantage approfondir les explications.

(19c) Il faut rester concentré.

(19d) Il faudrait s'impliquer un peu plus dans le travail de recherche.

(19e) Il faudrait simplement prendre davantage la parole en cours.

Dans les exemples (19d) et (19e), l'impersonnel et le mode conditionnel peuvent être considérés comme un double contournement de l'injonction frontale que représenterait l'usage du mode impératif.

-Utilisation de la périphrase verbale devoir + verbe (11\% des occurrences) :

(20a) Début d'année correct, vous devez intensiffer la participation.

(20b) Début d'année correct, mais vous devez vous impliquer davantage (...).

(20c) (...) mais vous devez vous investir en classe de facon régulière.

-Utilisation du type déclaratif :

(21) (...) mais l'attention n'est pas assez soutenue.

Dans l'exemple (21), le type déclaratif n'est pas simplement utilisé pour poser un constat. On peut en effet considérer que le locuteur, en utilisant le présent et non le passé composé, donne à sa phrase une valeur d'ordre indirect.

-Utilisation de l'infinitif précédé de la préposition à

L'emploi de l'infinitif au moment de faire une exhortation plus ou moins contraignante permet assez commodément d'effacer l'agent de l'action à réaliser comme on pourrait l'observer dans une annonce immobilière (dans des expressions telles que «à rafraîchir » ou «à rénover » par exemple).

(22a) Le travail reste à approfondir et la participation est à intensifier.

(22b) Elève assez sérieux, des bases à l'écrit et à l'oral à enricbir et à consolider, ayez une attitude plus concentrée.

On remarquera le tour assez peu naturel de l'exemple (22b) qui associe de manière intéressante l'infinitif avec effacement de l'agent et l'utilisation du mode impératif. Tout se passe comme si le locuteur avait consenti à alambiquer quelque peu son propos afin d'éviter le discours trop dirigiste qu'aurait été un «Cherchez à enrichir vos bases à l'écrit comme à l'oral. » et s'autorisait ensuite à revenir à l'impératif dans une formule où ce mode se voit doté d'une certaine souplesse. Le professeur applique en effet l'impératif «ayez » au nom «attitude », subtil dérivatif qui évite d'engager trop fortement la responsabilité du destinataire et rend l'impératif moins vigoureusement prescriptif que dans « Soyez plus concentrée. » 
Mais les stratégies d'effacement de l'interlocuteur ne passent pas uniquement par le discours injonctif. Si l'on quitte ce cadre, on s'aperçoit que le rôle d'actant de l'élève et même sa simple référence sont fréquemment effacés, notamment lorsque les constats sont assez délicats ou désagréables, ceci grâce à la forme passive ou à des constructions pronominales passives :

(23a) Programme non acquis ${ }^{14}$.

(23b) Des capacités parfois non exploitées.

(23c) Les efforts attendus n'ont pas été fournis. Les difficultés se sont accrues.

Mais on peut encore aller plus loin dans la recherche des procédés d'atténuation de la responsabilité de l'interlocuteur et évoquer dans certains cas un détournement de la notion même de sujet. Observons pour cela les exemples suivants :

(24a) (...) Début convenable mais qui ne vous a pas fatigué car votre travail est resté mesuré.

(24b) (...) les efforts n'ont pas abouti à une élévation conséquente des performances.

Dans l'exemple (24a), on peut considérer « début convenable » et « votre travail» comme des sujets postiches qui n'ont pas d'autre vocation que d'éviter de placer le pronom personnel vous en position avancée de sujet. Cantonnées à la fonction objet ou inscrites dans la détermination du nom, les marques de la deuxième personne n'ont ainsi qu'une visibilité moindre forcément moins impliquante pour l'interlocuteur mais aussi et surtout pour le locuteur qui évite d'engager de façon peut-être polémique la responsabilité de celui auquel il s'adresse dans les faits qu'il constate. L'exemple (24b) est quant à lui intéressant dans le sens où le sujet grammatical se trouve vidé de toute référence affective au sujet moral pour ne plus s'intéresser qu'à son substrat objectif enfermé dans le nom efforts, ici muni du seul article défini contenu dans le déterminant possessif (Wilmet, 1983) vos ou tes que l'on attendrait intuitivement.

On voit donc que le bulletin scolaire tend à produire une langue prudente, capable de réduire les affects potentiellement liés à l'évaluation. Tout semble fait pour que l'évaluation du travail soit perçue comme étant clairement distincte de l'évaluation des individus.

\subsubsection{Les destinataires indirects}

Ces destinataires indirects, non adressés mais marqués linguistiquement et reconnus par le locuteur, correspondent à ce qu'A. Rabatel (2010) appelle des «destinataires seconds intentionnels». Cette question des destinataires indirects est intéressante en tant qu'elle permet d'interroger la notion de routine. Ces destinataires indirects sont en effet plus particulièrement soumis à des variations culturelles et éventuellement historiques tandis que la place du destinataire direct s'impose presque comme une évidence.

\footnotetext{
${ }^{14}$ La dépersonnalisation que permet ce tour a justifié, semble-t-il, aux yeux du locuteur, l'éviction de la forme vous + verbe «Vous n'avez pas acquis le programme.» que nous jugeons pourtant plus naturelle.
} 


\section{(i) Le professeur s'adresse aux parents}

Dans ce cas, on parle alors de l'élève comme d'un absent, représenté ou non par un pronom personnel :

(25a) Bonne écoute quand le sujet l'intéresse.

(25b) Victoire s'est investie avec sérieux en badminton ce trimestre.

L'usage du prénom donne sans doute l'image d'une prise en compte plus personnelle, plus individuelle de l'élève qui vise peut-être à donner aux parents le sentiment que leur enfant est considéré dans sa spécificité la plus singulière ${ }^{15}$. Malgré cet effort de proximité, on s'aperçoit que la règle reste la distance et la prudence. La relégation des parents au rôle de destinataires indirects peut s'expliquer de deux façons : l'absence des marques d'adresse maintient tout d'abord les parents en dehors du «territoire» de l'école qui, de ce fait, reste symboliquement un lieu d'expression réservé aux seuls professionnels qualifiés. Mais cette exclusion peut également s'expliquer par l'engagement tacite pris par la communauté enseignante de ne pas mettre en cause la responsabilité des parents dans les faits qu'elle est amenée à constater. Ainsi, l'appréciation «Vous n'avez pas suivi le travail de Victoire » paraitrait-elle tout à fait audacieuse. A vrai dire, il semble possible d'imaginer que les deux phénomènes que nous avons mis en évidence soient étroitement liés : c'est parce que le professeur refuse l'ingérence des parents qu'il offre en contrepartie sa neutralité dès lors qu'il pourrait être question des choix éducatifs effectués par ceux-ci.

\section{(ii) Le professeur s'adresse à l'institution}

C'est principalement le cas des appréciations qui désignent le collégien ou le lycéen par le nom élève. Cette désignation ne semble en effet pas plus convenir à l'élève concerné (qui se perçoit en général comme un adolescent) qu’à ses parents (qui le perçoivent avant tout comme leur enfant). Cette désignation par le statut revêt en réalité un caractère académique qui laisse à penser que le locuteur se préoccupe de la visée diagnostique du document qu'il est en train d'élaborer, document qui ne serait donc pas uniquement perçu comme un outil de communication avec la famille et / ou l'élève. Ne l'oublions pas en effet, le bulletin est une pièce du dossier scolaire et peut, à ce titre, être étudié en commission de recrutement dans certaines formations sélectives (classes préparatoires par exemple) ou tout simplement en commission d'appel après une décision de conseil de classe litigieuse. Une telle utilisation du bulletin est assez régulière aux niveaux d'enseignement qui constituent des paliers d'orientation et que l'on qualifie plus banalement de classes à examen. Nous pensons à la classe de troisième et surtout à la classe de terminale. Les professeurs qui interviennent à ce dernier niveau savent et font savoir à leurs élèves que les bulletins peuvent être étudiés

\footnotetext{
15 Même si nous n'entendons pas approfondir cette question de l'usage du prénom, celle-ci ne nous parait pas tout à fait anodine. Dans un commentaire hippique tel que «S'il s'est surtout mis en évidence sous la selle cet hiver, il possède également de sérieux titres à l'attelage. ", on peut considérer que la mention du nom du cheval dont on parle laisserait une impression d'incongruité. Les raisons que l'on pourrait invoquer ici ne relèveraient pas tant de la nature animale du référent mais tiendraient plutôt à des enjeux relationnels totalement différents de ceux qui sont à l'œuvre dans le bulletin.
} 
par le jury du baccalauréat. Il n'est donc pas étonnant de constater que le professeur scripteur ait aussi le souci d'établir un compte rendu exploitable par les membres de l'institution qui pourraient un jour avoir besoin de consulter le diagnostic fait au sujet de celui qu'on désignera alors prioritairement comme un élève. Dans ce cas, l'évaluation prend une valeur, si l'on peut dire, de recommandation positive ou négative. Le bulletin scolaire devient dès lors une sorte de certification officielle de la valeur de l'élève destinée à ceux qui auront à le recruter ou à le juger dans un autre établissement.

\subsubsection{Le quatrième destinataire}

Il nous semble que l'on ne peut clore la question de la destination des propos sans évoquer la possibilité d'un quatrième destinataire. Afin de comprendre ce point de vue, il faut considérer le fait que l'enseignant écrit sous le regard de ses pairs dans le sens où ses propos ne bénéficient pas d'une parfaite confidentialité par rapport aux collègues qui enseignent aux mêmes élèves que lui. Même si la généralisation de la saisie numérique et la confidentialité des sessions informatiques qu'elle implique parfois peut limiter les possibilités de lecture des appréciations entre collègues, il n'en reste pas moins que le bulletin scolaire fait l'objet d'une lecture orale et parfois d'une vidéoprojection au conseil de classe qui ne manqueront pas d'exposer les choix des divers scripteurs. Ce regard collégial de la profession constitue sans doute, de manière plus ou moins lointaine, et de manière plus ou moins consciente, un sujet de préoccupation pour chacun des locuteurs dans lequel se trouve engagée une certaine image de soi. On peut ajouter que l'appréciation générale a ceci de particulier qu'elle est élaborée en vue du conseil de classe et soumise à l'approbation des collègues présents. Dans ce cas de figure particulier, celui qui a en charge la rédaction de cette appréciation (le professeur principal en règle générale) recherche explicitement la validation de son propos par ses collègues. Il doit donc à la fois proposer une appréciation lisible par les destinataires extérieurs et distants que nous avons évoqués plus haut et se conformer aux attentes immédiates et, si l'on peut dire, locales, d'un destinataire constitué d'individualités professionnelles. Dans cette dernière situation, la tentation du conformisme, que l'on peut considérer comme un effet indirect de la présence de ce quatrième destinataire, peut être particulièrement forte. Même s'il est difficile de dire dans quelle mesure la prise en compte du collègue destinataire est réellement consciente, il semble également difficile de nier totalement l'existence d'une interaction plus ou moins contraignante des différents scripteurs ${ }^{16}$. Cette interaction

16 Il semble précisément que l'abandon du tutoiement utilisé en classe au profit du vouvoiement pratiqué sur le bulletin peut relever d'une volonté de «lisser» la relation à l'élève, et ce afin d'en dissimuler les spécificités au moment où intervient le regard potentiellement juge des pairs. Il faut ajouter à cela le fait que ce vouvoiement ne semble pas favorisé par l'écrit mais bien par ce regard collégial de la profession que nous évoquons. En effet, l'examen des copies d'élèves révèle qu'un tutoiement pratiqué à l'oral est globalement maintenu à l'écrit. Le vous employé dans le bulletin scolaire ne s'adresse donc pas tant à l'élève (dont le tutoiement à l'écrit ne semble guère poser de problème) qu'à la cohorte de collègues que le scripteur institue mentalement comme des destinataires plus indirects. Cette remarque sur la pratique du vouvoiement nous semble importante car elle permet de constater que ce quatrième destinataire n'est pas si impalpable qu'il y parait et montre au contraire que l'on peut se fonder sur un argument linguistique pour légitimer sa place dans la problématique du rapport entre genre et grammaire. 
des scripteurs ne constitue d'ailleurs pas un trait distinctif du genre du bulletin puisqu'elle est également à l'œuvre dans le livre d'or. Avant de rédiger son appréciation personnelle, il n’est pas rare que le scripteur lise ce que ses prédécesseurs ont écrit. Cette lecture préalable l'influence, lui donne, d'une certaine manière, le ton de la communauté. Il ne faut en effet pas perdre de vue que, comme le bulletin, le livre d'or constitue une compilation, un édifice textuel bâti par divers auteurs. Dans cette configuration, chacun garde à l'esprit qu'il n'écrit pas seulement de manière horizontale mais aussi de manière, pourrait-on dire, verticale en tenant compte de ceux qui ont écrit ou qui vont écrire au-dessus et en dessous. Pour autant et quoi que l'on veuille bien considérer au sujet de la variété des destinataires dans le bulletin scolaire, la question de la destination du propos s'avère suffisamment flottante pour qu'on puisse voir les trois premiers modes d'adresse que nous avons évoqués apparaitre sur un même bulletin. Parfois, c'est à l'intérieur d'une seule et même appréciation que ces variations sont perceptibles comme nous l'avons vu dans l'exemple (22b) et comme nous allons le voir dans l'exemple (26) qui présente un cas flagrant de discontinuité allocutive entre la première et la deuxième phrase :

\section{(26) Elève travailleuse et sérieuse. Continue ainsi.}

Nous reconnaissons volontiers que la question du quatrième destinataire laisse planer quelques incertitudes : il est en particulier difficile de dire dans quelle mesure le locuteur a conscience des routines qu'il manipule ou qui le manipulent. Cependant, occulter cette question au nom de ces seules difficultés constituerait sans doute une erreur en tant qu'on manquerait alors de révéler une des caractéristiques essentielles du genre du bulletin qui se définit aussi par les incertitudes qui pèsent sur son statut et sa vocation à l'heure actuelle.

\section{Conclusion}

Par sa complaisance manifeste dans le figement et par toutes les stratégies qu'il met en place pour limiter les affects, le bulletin apparaît comme un genre qui tend, à proprement parler, à désincarner l'usage de la langue. Chaque locuteur impliqué s'efforce en effet de donner de l'évaluation scolaire une image raisonnée à travers laquelle la parole subjective du professeur n'émane finalement que pour se nier tant les choix linguistiques effectués rappellent que le fonctionnaire ne se sent autorisé à s'exprimer qu'en vertu de sa capacité à juger avec le plus haut degré d'objectivité et une pondération qui doit permettre à ceux qui font l'objet d'un jugement de se sentir appréciés avec justesse et distance. Mais ce qui peut relever d'un louable sens de la précaution oratoire peut également être interprété comme le signe d'une assez grande insignifiance pour ne pas dire tout simplement d'une banale langue de bois. On est en droit de se demander ici quel bénéfice réel peut tirer l'adolescent et sa famille du discours d'évaluation produit dans ces conditions. En accréditant l'idée que le bulletin est un genre, qui plus est routinier, on s'éloigne en effet considérablement de l'idéal d'une évaluation individualisée, fondée sur autre chose que des traits de signification minimaux (satisfaisant / insatisfaisant). 
On pourrait d'ailleurs finir comme l'on a commencé et se demander ce que l'expression "peut mieux faire » transmet réellement comme contenu sémantique et si elle n'est pas plutôt le signe d'un esprit indécis, réticent à juger vraiment et qui refuse de faire le «don» de sa subjectivité. Car ces étiquettes dont fait si souvent usage le bulletin scolaire peuvent aussi être perçues comme le signe d'une langue plus impersonnelle que réservée et se montrer peut-être révélatrices de rapports plus inquiets que prudents. Dans un contexte parfois tendu où l'exigence de respect ne semble pas toujours compatible avec la fermeté du jugement, et où la légitimité des statuts d'autorité est remise en cause, le locuteur choisit d'avancer en position retranchée. Quelquefois même, il procède à une véritable désertion énonciative, symbole non tant de son désengagement moral que de ce que l'on pourrait considérer comme une attente d'intronisation.

\section{BIBLIOGRAPHIE}

ADAM, J.-M., 2010, Les Textes : types et prototypes (édition revue), Armand Colin.

BOUQUeT, S., 2007, « Contribution à une linguistique néo-saussurienne des genres de la parole : une grammaire du morphème $\mathrm{ON} », \operatorname{LINX}$ n $^{\circ} 56$, Paris, Université de Paris XII Nanterre, p. 143-156.

Branca-Rosof, S., 1999, «Des innovations et des fonctionnements de langue rapportés à des genres », Langage et Société 87, p. 115-129.

Charaudeau, P. et Maingueneau, D., 2002, Dictionnaire d'analyse du discours, article "polémique », Ed. du Seuil, p. 437-438.

Maingueneau, D., 2004, «Retour sur une catégorie : le genre » dans Adam J.M, Grize J.B et Ali Bouacha M., Texte et discours : catégorie pour l'analyse EUD Dijon, p. 107-118.

KRAZEM. M., 2012, "Le Roman au secours de la phrase averbale », dans Liber aureus. Mélanges d'antiquité et de contemporanéité offerts à Nicole Fick, S. Laigneau-Fontaine, F. Poli (éds.), ADRA, p. 605-626.

LEFeuvre, F., 1999, La Phrase averbale en français, Lharmattan.

Moreau, M.-L., 1992, «Comment l'importance devient capitale » dans Hommage à Nicolas Runet, L. Tasmanowski et A. Zribi-Hertz (eds.), Communication et cognition, Ghent, p. 387-397.

Rabatel, A., 2010, "La Constellation des destinataires» dans Le Destinataire au théatre, Cl. Despierres et F. Fix (eds.), EUD, p. 13-24.

Rastier, F., 2001, Arts et sciences du texte, Paris, PUF.

WiLmET, M., 1983, « Les Déterminants du nom en français, essai de synthèse », Langue Française n 57 , p. $15-33$. 